\title{
Modelling deep indeterminacy
}

\author{
George Darby ${ }^{1} \cdot$ Martin Pickup ${ }^{2}$
}

Received: 18 July 2018 / Accepted: 21 February 2019 / Published online: 28 February 2019

(c) The Author(s) 2019

\begin{abstract}
This paper constructs a model of metaphysical indeterminacy that can accommodate a kind of 'deep' worldly indeterminacy that arguably arises in quantum mechanics via the Kochen-Specker theorem, and that is incompatible with prominent theories of metaphysical indeterminacy such as that in Barnes and Williams (Oxf Stud Metaphys 6:103-148, 2011). We construct a variant of Barnes and Williams's theory that avoids this problem. Our version builds on situation semantics and uses incomplete, local situations rather than possible worlds to build a model. We evaluate the resulting theory and contrast it with similar alternatives, concluding that our model successfully captures deep indeterminacy.
\end{abstract}

Keywords Metaphysical indeterminacy $\cdot$ Deep indeterminacy $\cdot$ Kochen-Specker . Situation semantics $\cdot$ Indeterminacy

\section{Introduction}

This paper presents a model of metaphysical indeterminacy. It attempts to provide a structure that captures the sort of 'deep' metaphysical indeterminacy which causes problems for a number of existing theories. Our core idea is this: metaphysical indeterminacy is a matter of reality being unsettled between parts which fundamentally disagree about how the world is.

Providing models for metaphysical indeterminacy is a flourishing industry (see, e.g. Akiba 2004; Barnes and Williams 2011; Wilson 2013; Torza 2017; Calosi and Wilson 2018). The motivation is to provide a systematic account of how metaphysical indeterminacy should be theorised. This is valuable because to investigate metaphysical indeterminacy and its applications thoroughly, we should first be clear about how to understand it. Even the sceptic about the existence or coherence of metaphysical indeterminacy should welcome such clarity.

\footnotetext{
Martin Pickup

martin.pickup@philosophy.ox.ac.uk

1 Oxford, UK

2 Oriel College, Oxford OX1 4EW, UK
} 
Several such theories of metaphysical indeterminacy (Akiba 2004; Barnes and Williams 2011, for example) share a common feature-they presuppose that although reality is not precise, it could be. Metaphysical indeterminacy is then explained as unsettledness between different precise candidates for how reality is. Quantum indeterminacy is especially interesting in this regard (Darby 2010; Skow 2010), because although it is one of the main naturalistic motivations for a theory of metaphysical indeterminacy, the reason that it is a popular example of genuine metaphysical indeterminacy is that quantum reality simply cannot be precisified all at once-the Kochen-Specker theorem appears to show that assigning precise values to all observables is mathematically impossible (given non-commutative algebras of observables). Thus the theories of metaphysical indeterminacy that require complete precisifications of reality are undermined.

We present what we consider an obvious solution: to work with a view according to which precisifications are local rather than global. Kochen-Specker rules out assigning precise values to all observables, so a natural fix is for a theory of metaphysical indeterminacy to assign precise values to only some observables in this context. The move parallels the thought that although the metaphysics literature makes great use of possible worlds, which are essentially complete or maximal, there are many purposes, among which is this one, for which less-than-maximal possibilities are theoretically important. This idea can be cashed out in various ways, notably in situation semantics, which uses parts of possible worlds (situations) as the basis of meaning. Situations are thereby inherently partial (Kratzer 1989), and various theoretical advantages are claimed including accounts of anaphora (Elbourne 2005), definite descriptions (Elbourne 2013) and the liar paradox (Barwise and Etchemendy 1987). ${ }^{1}$

Our approach will be to take incompatible situations as our candidate precisifications between which reality is unsettled. We explore how these devices are naturally given a metaphysical reading in the style of Barnes and Williams (2011). In doing so, we propose an account of metaphysical indeterminacy which neatly aligns with quantum mechanical motivations for understanding reality as unsettled.

Our paper will be structured as follows: first we shall give some brief background on existing accounts of metaphysical indeterminacy. We will then explain the distinctive challenge of 'deep' indeterminacy (in the quantum case and in general). Our fourth section introduces our model in some detail. In our fifth section we evaluate the model, especially with respect to some nearby alternatives we have chosen not to pursue, before concluding.

\section{Meta-level accounts and the Barnes-Williams model}

To present our alternative, we should first give a brief recap on the state of play.

\footnotetext{
1 Situations are also used by Edgington (1985) in the context of Fitch's Paradox and Rumfitt (2015 and elsewhere) in the context of logical consequence, to give further examples.
} 
What is indeterminacy? One helpful way to think of it is as an irremovable sort of unsettledness. It has a number of different species. A paradigm understanding of indeterminacy is found in the vagueness literature in the philosophy of language: it is indeterminate whether some stones make a heap. This kind of indeterminacy is naturally thought of as representational: it is not 'out there in the world', rather there is just no fact of the matter about whether those stones fall under the meaning of the word 'heap'. The indeterminacy involved in these vagueness cases is therefore naturally interpreted as semantic.

Metaphysical indeterminacy is indeterminacy of a different sort. The intuitive idea of metaphysical indeterminacy is that it is non-representational indeterminacy. This requires elaboration. The core notion is that metaphysical indeterminacy is indeterminacy that is really 'out there in the world'. If semantic indeterminacy is a result of some sort of deficiency in language, and epistemic indeterminacy is a result of deficiency in knowers, metaphysical indeterminacy in a result of deficiency in how things are. Along these lines, Barnes (2010) takes metaphysical indeterminacy to be that indeterminacy that would remain even if the all the representational indeterminacy were removed - that is, if all other sources of deficiency were taken away.

Saying what metaphysical indeterminacy is supposed to be is obviously also relevant to whether there is, or could be, any. This issue is contentious: it is by no means universally accepted that the notion of metaphysical indeterminacy is coherent. Nor is it universally accepted that metaphysical indeterminacy is possible. One indirect way to argue for the coherence and possibility of such indeterminacy is to construct explicit models of it; providing precise accounts of a purported phenomenon can make that phenomenon seem more respectable. Examples of models of metaphysical indeterminacy include Akiba (2004), Barnes and Williams (2011), Wilson (2013) and Torza (2017). Here we focus on a particular class of models which treat metaphysical indeterminacy in a way akin to linguistic indeterminacy. In a case of linguistic indeterminacy, language could be more precise, but is not. Indeterminacy arises when something is true on some precisifications of the language and false on others. In a parallel way, these models take it that the world itself could be more precise, but is not. Metaphysical indeterminacy then arises when something is true on some precisifications of the world and not on others. This class of models are called 'meta-level' accounts by Wilson (contrasting with her own 'object-level' view).

The main idea in Barnes and Williams (2011) ('BW') is that we should treat metaphysical indeterminacy in much the same way as the supervaluationist treats cases of vagueness: namely by considering different candidate precisifications and regarding something as indeterminate when the different precisifications disagree about that thing. To be more specific, BW propose a model whereby there are different candidate precisifications of actuality. These precisifications are ersatz possible worlds, and to be a candidate for representing actuality is to not determinately fail to represent actuality. So the ersatz worlds are representations of reality, and some group of them are distinctive by being those which do not determinately represent actuality inaccurately. These worlds, which are complete and precise, are the mechanism by which to express indeterminacy. It is determinate that $\mathrm{p}$ iff all of the ersatz worlds that are candidates for representing actuality are p-worlds, determinate that $\neg \mathrm{p}$ iff none of these worlds are p-worlds, and indeterminate whether $\mathrm{p}$ iff some but 
not all of these worlds are p-worlds. So, formally speaking, we evaluate propositions not at a specific (maximal, sharply defined) world, but rather evaluate them on sets of worlds. If there is no metaphysical indeterminacy, only one candidate will not determinately misrepresent reality, and so the model will collapse into the familiar framework.

(Because they use 'not determinately failing to represent actuality' as the condition for being a member of the set of precifications, the Barnes-Williams model is not reductive in an important sense. But our main focus here-the source of the trouble with deep indeterminacy and the reason for the move from worlds to situations - is on the structural features of the model rather than its reductive ambitions.)

BW add to this structure the claim that "there is the One True actualized worldit is just indeterminate which world this is" (p. 131). This can be a little hard to parse, but we understand it to mean that there really is exactly one ersatz world which correctly represents actuality, but it is indeterminate which one it is.

A feature of the BW model is that it can uphold classical logic (BW point to this as a benefit). In particular, both bivalence and excluded middle are retained. They have bivalence because every possible world which is a candidate is bivalent: for any proposition $\mathrm{p}$ either $\mathrm{p}$ is true in a world or $\mathrm{p}$ is false in that world. Given that these are the candidates for actuality, and that one of them is in fact the One True world, bivalence holds. The theory retains the principle of excluded middle because for any proposition $\mathrm{p},(\mathrm{p} \vee \neg \mathrm{p})$ is true at all worlds, so determinately true.

On the BW approach, indeterminacy has an interesting parallel with modality. Something is necessary when true in all possible worlds and contingent when true in some but not all possible worlds. Similarly, on the BW model, something is determinate when true in all the possible worlds in a restricted range and indeterminate when true in some but not all of the possible worlds in that range. The relevant possible worlds are those which do not determinately fail to represent actuality. As a consequence, a disjunction might be determinate while its disjuncts are indeterminate, in the same way that a disjunction can be necessary while its disjuncts are contingent. For instance, 'the university is in the city centre' and 'the university is in the suburbs' may both be indeterminate while 'the university is either in the city centre or in the suburbs' is determinately true.

The modal comparison will be significant later when we consider the needed modification to cope with deep indeterminacy-the modification is analogous to treatments of modal logic using less-than-maximal possibilities instead of the more familiar possible worlds. But first, we shall set out the issue arising for the BW model from deep indeterminacy.

\section{The challenge of deep indeterminacy}

As has been mentioned above, quantum mechanics is often cited as an example of metaphysical indeterminacy, or indeed as a motivation for developing a theory of such indeterminacy. Many more recent interpretations of quantum mechanics, 
however, don't obviously seem to generate a case for such indeterminacy. ${ }^{2}$ Nevertheless, and without going into the interpretative assumptions that are required to underwrite a move from quantum mechanics to worldly indeterminacy, it is commonly claimed that that there is at least motivation from physics for seeking an account of metaphysical indeterminacy (see, for instance, Williams 2008; Wilson 2013; and Bokulich 2014).

Although we think it can be doubted that quantum mechanics gives us motivation to seek a model of metaphysical indeterminacy, what we are interested in here is a different sort of problem. The problem is the following: many of those who present quantum mechanics as a motivation for accepting genuinely metaphysical indeterminacy provide models which aren't able to capture precisely the quantum mechanical phenomena they point to as a motivation. Following Skow, we'll focus on the Barnes-Williams model, but this issue plausibly arises for others too. The issue for BW is that the candidates for actuality in their model, the candidates between which reality is unsettled, are ersatz possible worlds. But worlds are maximal; being a possible world involves settling everything. In particular, every observable gets a value in a possible world. But the essence of the Kochen-Specker result is that it is impossible for every observable to have a value at once. It doesn't even make sense within the mathematical constraints of the formalism to claim that every observable gets a value in the relevant contexts. Thus this kind of 'deep' indeterminacy cannot be modelled by the Barnes-Williams framework because the tools they use for the modelling are complete and precise.

But what exactly is 'deep' metaphysical indeterminacy? Skow uses the term to refer to "metaphysical indeterminacy [which] runs so deep (or can run so deep) that reality cannot be completely precisified." (Skow 2010, p. 852, italics in original) The quantum mechanical case is therefore an alleged instance of this sort of metaphysical indeterminacy. Deep metaphysical indeterminacy is thus the sort of indeterminacy which resists complete precisifications, even into alternatives. It renders reality by nature incomplete.

A related notion of deep indeterminacy is also worth mentioning. This takes deep metaphysical indeterminacy to be indeterminacy which is prescribed by the nature of the domain under discussion. It is part of the theoretical set-up that there is indeterminacy involved; indeterminacy is not an optional extra. In other words, any representation of the domain which is not indeterminate cannot be an accurate representation of how the domain could be: to fail to be indeterminate is to determinately

\footnotetext{
${ }^{2}$ For example, in the modern Everett interpretation the underlying ontology, from which the branches emerge, is perfectly determinate (whether the branches themselves are is another matter, but the underlying ontology is the level at which one would naturally judge the question of whether there is metaphysical indeterminacy). In the modern 'flashy' GRW theory, the distribution of flashes in spacetime is also perfectly determinate. If one subscribes to the view that, one way or another, what is fundamental is the wave function evolving in configuration space then yet again the fundamental ontology is perfectly determinate. Perhaps there is a sense in which derivative ontology that does include indeterminacy still falls under what is intended by 'metaphysical indeterminacy', but this is much less clear. See Glick (2017) for discussion: he is sceptical that there is a motivation to be found here.
} 
fail to represent the relevant actuality. ${ }^{3}$ The quantum mechanical example is also an alleged case of this more general view of deep indeterminacy.

The key point is that, in both senses, deep metaphysical indeterminacy cannot be modelled as unsettledness between complete, precise alternatives: these alternatives cannot be complete and precise if they are really to be alternatives for how things in fact are. So, independent of the precise quantum mechanical motivations for seeking a theory of metaphysical indeterminacy, we think it worth providing an account of metaphysical indeterminacy which allows for its deep variety.

The response we'll pursue is to show how one can model deep indeterminacy by adjusting the BW framework in ways that we take to be independently plausible. Before we spell out the model, however, we should briefly note a dialectical point given our bracketing of various other potential responses to the issues arising from the Kochen-Specker result.

We don't wish to follow Skow in claiming that the Kochen-Specker result shows that deep indeterminacy is clearly possible, exactly, but just that it is at least important to explore how theories of metaphysical indeterminacy should handle it. There are coherent interpretative assumptions which would render quantum mechanics a case of deep indeterminacy. Even if the best interpretation of quantum mechanics does not turn out to warrant these assumptions, now that we've become aware of the possibility of deep indeterminacy it worth investigating how the Barnes-Williams model can be revised to account for it, given that it is at present incapable of doing so. Deep metaphysical indeterminacy sounds, on the surface at least, like it should be possible if the very idea of metaphysical indeterminacy is, and so should be accounted for by an acceptable theory.

\section{Modelling deep indeterminacy}

\subsection{Situations and indeterminacy}

Our solution to the problem of deep indeterminacy will involve using different theoretical machinery. We will use situations to model metaphysical indeterminacy in a way that makes space for deep indeterminacy. ${ }^{4}$ As a first gloss, situations can be introduced as parts of possible worlds. This is only a gloss, though, as a thoroughgoing situation theorist will prefer to take situations as the theoretical primitive and then (if necessary) define possible worlds as special sorts of situation. How, exactly,

\footnotetext{
3 This notion can be applied more generally. As an example, we, along with Jon Robson as a co-author, discuss deep indeterminacy of this sort in the context of fictional indeterminacy in Darby, Pickup and Robson (2017).

4 What follows uses the formal machinery of situation semantics. There are other semantics akin to situation semantics which might be conducive to a similar approach. In particular, Fine's Truthmaker Semantics (Fine 2017) might be a fruitful alternative way to express the metaphysical claims here advanced (see also Yablo 2014 for discussion of 'aboutness'). What will be crucial is the partial nature of the entities under consideration and the denial of monotonicity that occurs below relating to persistence.
} 
to fully characterise situations isn't a settled issue in situation theory. ${ }^{5}$ Nevertheless, whatever they are, situations are paradigmatically partial: they do not have to tell us everything. ${ }^{6}$ For instance, the situation encompassing only World War 2 does not give us any information about World War 1, or future Martian settlers, or events happening on a distant planet in 1945. To put it another way, situations will give answers to some, but not all, questions (whereas worlds give an answer to every question). Situation semantics proposes that we evaluate propositions with respect to situations rather than worlds. While possible world semantics offers possible worlds as the entities with respect to which propositions are evaluated, situation semanticists simply replace these maximal worlds with partial situations.

There are several advantages to evaluating with respect to situations rather than worlds. ${ }^{7}$ But we do not wish to be committed to situation semantics per se. Rather, we want to see what metaphysical work the tools of situation theory can do in the present context. Given this purpose, we will only introduce what machinery is necessary to do this work. Specifically, although there are a variety of ways to flesh out a situation theory and corresponding semantics, we will only fill in the picture where it is required for presenting our model.

The proposition expressed by a typical statement like 'Jane is swimming' will be evaluated with respect to some particular situation: following Austin we take the statement to give both a claim and a specific situation with respect to which we can evaluate the claim. ${ }^{8}$ The proposition is true if Jane is swimming in the relevant situation. The proposition is false if Jane is, say, running in the relevant situation. But not all situations of evaluation will settle the question of whether Jane is swimming: situations that don't contain Jane, for instance, will not say whether she is swimming. There is a situation-theoretic choice to make about the truth-value of the proposition in such situations. One alternative is to take the proposition to be false with respect to situations which don't address the claim that is made. The other is to take the proposition to be neither true nor false. We favour the latter view, and this will be important for our model.

Why believe that a proposition is neither true nor false when the situation of evaluation doesn't settle the issue? Without going into too much detail, there are a few indicative reasons. One is that situations, being partial, just don't tell us everything. If we take proposition to be false in situations which don't settle the question of Jane's swimming, we wouldn't be able to distinguish by truth-value situations which

\footnotetext{
5 Barwise (1988) lists a series of choice points for the nature of situations and their behaviour.

6 One might define possible worlds as special sorts of situation that are maximal in some sense. Such situations would not be partial in that sense. There might also be an unrestrictedly maximal situation that described everything that is possible (akin to the Lewisian pluriverse): this would not be partial in any sense. Whether there are possible worlds or a maximal element are open questions in situation theory, but the point here is simply that situations do not need to be maximal in any sense.

7 Kratzer (2017) describes some areas within semantics where situations have been used productively, including anaphora and the Liar paradox.

8 Broadly speaking, the claim is given by the 'type' of the statement, while the situation of evaluation is given by the particular 'stating' in context. In Austin (1950), which is a foundational text for this semantic approach, these are provided by two sets of conventions, the descriptive and the demonstrative respectively.
} 
show Jane is not swimming from those which are simply silent on the issue. Furthermore, if propositions are false in situations which don't settle the relevant question then incompatible statements like 'Jane is in motion' and 'Jane is at rest' will receive the same truth-value (namely 'false') in situations which, for instance, just don't contain Jane. ${ }^{9}$

This gives some motivation for the idea that propositions can lack truth values in situations. The example we have been using is a proposition about Jane's activity being evaluated with respect to a situation in which she is not present. More generally, propositions about individuals will typically be neither true nor false in situations which lack them. (This won't be the case for propositions asserting the existence of an individual, which will be false in situations lacking those individuals). ${ }^{10}$ This is not the only way to get a truth-value gap, however. Situations are finegrained, so that a situation which contains an individual need not contain all the predicates true of that individual. For instance, a situation in which Jane is swimming might not include the fact that Jane is thinking about her dinner plans. The proposition 'Jane is thinking about dinner' can therefore still come out as neither true nor false when evaluated with respect to a situation containing Jane, if that situation is appropriately restricted.

Propositions lacking truth-values in cases like this might be broadly described as those which are not about the situation (or, to put it the other way around, such situations underdetermine the propositions). This accords well with the spirit of situation theory: the fact that situations are partial is in sympathy with the view that situations don't give a truth-value for every proposition. However, we will require truth-value

\footnotetext{
9 There are ways to avoid this. One option is to restrict to atomic propositions: only atomic propositions which are not settled by a situation are false in that situation. If 'at rest' is defined as 'not in motion' and if 'Jane is not in motion' is equivalent to 'it is not the case that Jane is in motion', then 'Jane is at rest' will be the negation of 'Jane is in motion'. The atomic 'Jane is in motion' will be false and so its negation, 'Jane is at rest', will be true. This is sort of move invokes negative free logic.

There are reasons we are wary of this in the context of situation semantics. One is that it appears to rule out atomic propositions being incompatible. Though this is contentious, 'The apple is red' and 'The apple is green' appear to both be atomic and to be incompatible. Secondly, the equivalence of 'Jane is not in motion' and 'it is not the case that Jane is in motion' might be challenged in circumstances where Jane does not exist.

At any rate, these considerations were only supposed to be indicative of motives for taking some propositions to be neither true nor false: we are not wedded to them. Nor are we claiming that there is no plausible account on which a proposition is false when the situation doesn't speak to the proposition. (Thanks to a reviewer for prompting reflection on this.).

10 Why will it be false? Because such propositions assert of an individual that it exists, when there is no such individual in the relevant situations of evaluation.

A typical semantics will make an existential quantification of the form ' $a$ exists' true when and only when there is an admissible value of $x$ which can be substituted into the formula $x=a$ to give a true identity. What is an admissible value will depend on context: for situation theory a natural domain for possible values of $x$ is the individuals in the situation of evaluation. A situation in which $a$ doesn't exist will therefore not have an admissible value of $x$ which can make $x=a$ true. The statement ' $a$ exists' will therefore be false in such situations. See the Appendix to Pickup (2016) for a sketch of an example semantics.
}

Thanks to one reviewer for spotting that existential statements will be atypical in this way, and to both reviewers for prompting the more general discussion here. 
gaps in further cases as well. In particular, we'll claim propositions can be neither true nor false when situations overdetermine propositions. This will become clearer when put into practice, but the motivating idea is that certain situations fail to settle certain propositions when they are conflicted about those propositions. ${ }^{11} \mathrm{We}$ grant that cases like this might appear odd. Rather than defend them in the abstract, we hope that presenting the overall picture will be reassuring about the overall consistency of the model we present (even if it doesn't yet fully convince).

Our situation semantics, therefore, will allow propositions to lack truth-values, and in different ways. Two further, and uncontroversial, aspects of situation theory can be added. Firstly, that situations can be parts of one another (i.e. there are mereological relations between situations), and secondly that the evaluation of truth-values is situation-relative. This provides a relation between situations and a situationtheoretic account of truth. Having given the basic components of situation theory, we can formulate our preferred model of metaphysical indeterminacy.

\subsection{The model}

As noted, the trouble with the BW model is that it requires complete precisifications (in the form of entire possible worlds) as the candidates between which the world is not settled. In short, as mentioned above, situations can be used as precisifications of incomplete things. According to us, therefore, determinacy is a matter of truth in situations which are candidates for actuality.

To be explicit: we propose to replace the original BW structure with a new structure. BW evaluate propositions with respect to sets of ersatz possible worlds. These possible worlds are the candidate precisifications of reality. For BW, it is indeterminate whether $\mathrm{p}$ iff some of the ersatz worlds that are candidates for representing actuality are p-worlds, and some are $\neg$ p-worlds. It is determinate that $\mathrm{p}$ iff all these worlds are p-worlds. To be a candidate for representing actuality is to not determinately fail to represent actuality. Our structure evaluates propositions with respect to sets of (sharply defined but typically local and incomplete) situations. These situations are the candidate precisifications of parts of reality. A first pass at a situationtheoretic version of BW might be:

\section{Determinacy A proposition $\mathrm{p}$ is determinate iff it is true in every situation which is a candidate for representing reality \\ Indeterminacy A proposition $\mathrm{p}$ is indeterminate iff it is true in some but not all situations which are candidates for representing reality}

\footnotetext{
11 Deep indeterminacy, we'll claim, can be modelled as an instance of this. Kit Fine's Fragmentalism (2005) can also be interpreted in a cognate way, as generating conflicting fragments (= situations?) from the reality of tense. On one interpretation of Fine's view, different times will give different truth-values for propositions, leading to truth-value gaps in the 'über-reality' which contains multiple times. Other applications of this overdetermination approach include puzzles about the persistence of material objects (see Pickup 2016 for an application to the Ship of Theseus case).
} 
But this isn't quite right. For situations are partial, and will not contain all determinately true propositions. For instance, suppose it is determinate that a ball has a certain mass. The proposition expressing this will not be true in all situations which might represent reality, for many of these will simply not contain the ball. This is where our choice in the previous section to allow propositions to lack truth-values in situations starts to make a difference. For in situations which lack the ball, the proposition that it has a certain mass will be neither true nor false. Whereas a case of indeterminacy is a case where the different candidates will genuinely disagree about how reality is, i.e. the relevant proposition will be true in some situation and false in others. We can therefore offer the following definitions on our model:

Determinacy A proposition $\mathrm{p}$ is determinate iff it is true in some situation which is a candidate for representing reality and false in no such situation

Indeterminacy A proposition $\mathrm{p}$ is indeterminate iff it is true in some situation which is a candidate for representing reality and false in some other such situation

This captures the fact that indeterminacy isn't a matter simply of incompleteness, but rather of disagreement.

To see how this works in practice, we will take a determinate case, a typical case of indeterminacy and a case of deep indeterminacy and show how the definitions apply.

Let's assume it is determinate that a particular ball has mass M. How does this fit the model? There will be a number of different situations which fail to determinately misrepresent reality: this is our set of situations with respect to which we evaluate. Some of these will contain the ball with its mass, and some will not. The situations lacking the (whole) ball and its mass will give no truth-value to the proposition expressing the claim that the ball has mass $\mathrm{M}$ : it will be neither true nor false in these situations. The situations containing the (whole) ball will be ones in which it has mass $\mathrm{M}$, and so the relevant proposition will be true in them. This exhausts the situations in the evaluative set. Thus the proposition will be determinate: it is true in at least one situation which is a candidate for representing reality and false in no other such situation.

Next, suppose it is (metaphysically) indeterminate whether Jones has some property F. Within the set of situations which don't determinately misrepresent reality we will find many which say nothing about Jones's F-ness. In these, the proposition expressing that Jones is F will be neither true nor false. As it is indeterminate whether Jones indeed is F, the remaining situations in the set will be of two types. In some, Jones will be F. In others, Jones will be not F. Thus, in some situations which are candidates the proposition will be true while in others it will be false, not merely neither true nor false. (This requires some semantic assumptions according to which $\neg$ p being true entails $\mathrm{p}$ being false.) The proposition is therefore indeterminate, because it is true in some candidate situations and false in others. This works in much the same way as the supervaluationist approach to vagueness and, of course, in the way BW describe for worlds. 
Finally, suppose we have a case of deep indeterminacy. ${ }^{12}$ For ease, let's use a quantum mechanical case (granting the required interpretative assumptions). Again, there will be many situations in the set of candidates for representing reality which say nothing about this quantum system, and hence in which propositions relating to it are neither true nor false. But there will also be other sorts of situation. Let's consider four in particular, which contain certain information about the properties of the system. The first, $\mathrm{s}_{1}$, might, for instance, have a particle spin-up in the x-direction. Let this information be captured by a proposition $\mathrm{p}$. Thus, in $\mathrm{s}_{1}, \mathrm{p}$ is true. The second situation, $s_{2}$, is one in which the quantum system has contrary properties, e.g. the particle has opposite spin in the same direction. Thus, in $s_{2}$, the proposition $\neg p$ is true and so, given some semantic assumptions, $\mathrm{p}$ is false. So far, nothing differs from a normal case of indeterminacy, which can equally be captured by the BW model. But here's the distinctive advantage of situations: not all of the properties of the quantum system need to be precise in $\mathrm{s}_{1}$, which need for example include nothing about spin of that particle in the y-direction. Now take two further situations, $s_{3}$ and $\mathrm{s}_{4}$. In $\mathrm{s}_{3}$, some proposition $\mathrm{q}$, corresponding to some value for spin in the y-direction, is true, and $\neg q$ is true in $s_{4} \cdot{ }^{13}$ Because of the Kochen-Specker theorem, there is a mathematical constraint that no situation contains determinate truth-values for both $\mathrm{p}$ and $\mathrm{q}$. But this is not violated by $\mathrm{s}_{1}-\mathrm{s}_{4}$, as we can hold $\mathrm{q}$ to be neither true nor false in $s_{1}$ and $s_{2}$. And $\neg q$ will likewise lack a truth-value in $s_{1}$ and $s_{2}$. Similarly, both $\mathrm{p}$ and $\neg \mathrm{p}$ will fail to have a truth-value in $\mathrm{s}_{3}$ and $\mathrm{s}_{4}$. Thus in none of $\mathrm{s}_{1}-\mathrm{s}_{4}$ is there conflict with deep indeterminacy. We are not required to give truth-values to any pair-wise combination of, one the one hand, $p$ and $\neg$ p and, on the other, $q$ and $\neg q$.

Let's recap. We have four situations, and, in each, one of a pair of propositions is true (and the other false) while a second pair receives no truth-value. These situations are the candidate precisifications that give rise to the indeterminacy of the world with respect to the propositions. This, in essence, is our way to capture deep indeterminacy within a BW-style framework. By using partial things, namely situations, in the model we can make space for deeply indeterminate propositions.

So far, so good. But there is a sting in the tail. The situations which are candidates precisifications of actuality fundamentally disagree in cases of both gardenvariety and deep indeterminacy. There is a threat that this fundamental disagreement will lead to a contradiction if we are not careful. In the Jones case, for instance, there are situations in which Jones is F and situations in which Jones is not. If there are larger candidate situations containing both of these, and if what is true in a part of a situation is true in the whole situation, then the larger candidate situation will be contradictory: in it Jones will be F and not F. We therefore need to spell out how the fundamental disagreement necessary for indeterminacy can be maintained without falling into contradiction.

\footnotetext{
12 The example we use here is taken from our earlier joint work with Jon Robson (2017).

13 We are suppressing some details here, as in the quantum mechanical case there'd also be further situations corresponding to spin in the z-direction. Two pairs suffice for the demonstration, however.
} 


\subsection{Disagreement without contradiction}

Not all truths which are in candidate situations can be consistently combined. We need to provide a restriction that rules out a situation which is a candidate for actuality from containing a contradiction while maintaining the fundamental disagreement in the model. There is a choice to be made about what the restriction is a restriction upon. Three possibilities present themselves.

Firstly, the restriction could be a restriction on composition. According to this option, some situations compose a further situation only under some restricted circumstances. This solves the problem by finding an appropriate restriction such that there simply is no situation which contains as parts both a situation in which Jones is $\mathrm{F}$ and a situation in which Jones is not F. There would then be no threat of contradiction in any situation. Although situation theory usually operates with unrestricted and unique fusion as a mereological principle (at least for actual situations, on which more below), there's no reason in principle why this couldn't be rejected.

Secondly, the restriction could be a restriction on what counts as a candidate for actuality. According to this option, only in restricted circumstances does fusing two situations which are candidates for actuality result in a situation which is also a candidate for actuality. This solves the problem because, given an appropriate restriction, although the situations in which Jones is $\mathrm{F}$ and in which Jones is not $\mathrm{F}$ are each candidates for actuality, the situation which combines them is not. There is a connection with the previous possibility here, as in contexts without indeterminacy the only candidates for actuality will be the actual situations.

Thirdly, the restriction could be a restriction on how truth transfers between situations related by parthood. In situation theory this is referred to as the question of the persistence of propositions across situations. Universal persistence states that whenever a proposition is true in a situation which is a part of another, it is also true in the larger situation. Situation semanticists are divided about whether or not universal persistence should be accepted. ${ }^{14}$ A restriction on persistence would solve the problem by finding an appropriate restriction such that propositions about Jones's having or lacking $\mathrm{F}$ don't persist into the fused situation.

Broadly speaking, the choice of what to restrict upon is a choice between a mereological restriction (restricted composition), a modal restriction (restricted actuality) and a semantic restriction (restricted persistence). However, this choice will not make a difference to the formal task of providing a model for the account of metaphysical indeterminacy we propose. We will choose to take the semantic route and restrict which propositions persist from situations to their extensions for ease of exposition. We take our solution, however, to be translatable into the mereological or modal versions without issue.

\footnotetext{
14 For instance, Kratzer (1989) keeps hold of universal persistence, but Elbourne (2005, 2013) rejects it. One initial reason to reject it comes from universally quantified statements (where they can be true in small situations but false in larger ones containing the former).
} 


\subsection{A restriction on persistence}

As mentioned above, it is a contentious issue in situation semantics whether propositions universally persist. We will propose that, in certain contexts, they do not. Although we think a precise restriction on persistence might be formalised, so that propositions persist unless certain conditions are satisfied, for our purposes here it is not necessary to elaborate and defend such a restriction. ${ }^{15}$ What matters for the argument is to explain how the failure of universal persistence allows for an adequate account of metaphysical indeterminacy.

So, instead of providing a detailed account of restricted persistence we will operate with a guiding principle in line with the way we introduced truth-value gaps in Sect. 4.1 above. There we noted that some propositions can be underdetermined and some overdetermined by situations. Our guiding principle will be that propositions fail to persist when they are overdetermined, i.e. when some other part of the larger situation into which they would persist already says something about the proposition. This overdetermination can be cashed out in terms of preclusion. A proposition is precluded by a situation when the proposition cannot be true with the contents of that situation. ${ }^{16}$

It might be helpful to give a brief example. Imagine that a banana changes from being green to being yellow. The banana will be green in some situations at the earlier time and yellow in some situations at the later time. Suppose there is a larger situation $\mathrm{s}$ which has both an earlier and a later situation as parts. If propositions universally persisted, the proposition that the banana is green and the proposition that it is yellow would both be true in s. But the idea we're pursuing says that neither proposition persists, because the larger situation s overdetermines the colour of the banana. The proposition that the banana is green doesn't persist from the smaller situation at the earlier time into s because s contains a part (viz. a situation concerning the later time) which says something about the proposition that the banana is green. Specifically, the later situation precludes the proposition that the banana is green. Parallel reasoning applies to the proposition that the banana is yellow. Hence neither of these propositions persists into s. ${ }^{17}$ This should give an indication of how the machinery is supposed to work, which we can then deploy to address the current problem.

The problem we face is this: to explain how in a case of indeterminacy we get disagreement without contradiction. In short, the answer is that there are truthvalue gaps of the overdetermination character when propositions fail to persist in

\footnotetext{
${ }^{15}$ See Pickup (2016) sect. 2.3 for an earlier discussion of a restriction on persistence. The restriction there employed goes via counting of existential situations for the particulars referred to by the proposition. (An existential situation for some $a$ is a situation in which $a$ exists.) This paper does not rely on that account of restricted persistence.

${ }^{16}$ Of course, a full theory of preclusion would be necessary to give a precise account of this restricted persistence condition. As we have said, we do not intent to furnish such a complete account: this would be a different and much longer paper. But see the Appendix to Pickup (2016) for some groundwork.

17 This is clearly just a toy example, and much more would need to be said about it.
} 
indeterminacy cases. There is disagreement between situations but gaps, rather than contradictions, within situations.

Consider the situations containing Jones being $\mathrm{F}$ and Jones being not $\mathrm{F}$. The fusion of these situations is causing the problem, as if universal persistence were to be straightforwardly upheld this situation would have both 'Jones is F' and 'Jones is not F' true in it. Our suggestion is simply that both of these propositions lack a truth-value in the fused situation. This is because there is overdetermination of propositions about Jones's F-ness in the fused situation. ${ }^{18}$

We used situations to explain how the deeply indeterminate quantum mechanical case might be dealt with: in $s_{1} p$ is true, in $s_{2} p$ is false, in $s_{3} q$ is true and in $s_{4} q$ is false without those situations specifying any other truth-values. We can now show how contradiction is avoided in larger situations containing some of $s_{1}-s_{4}$ as parts. In any situation containing both $s_{1}$ and $s_{2}$ as parts, $p$ lacks a truth-value because $p$ is overdetermined (and likewise with $q$ in situations containing $s_{3}$ and $s_{4}$ ). The proposition $\mathrm{p}$ is precluded by $\mathrm{s}_{2}$ and $\neg \mathrm{p}$ is precluded by $\mathrm{s}_{1}$, so neither are true in the larger situation containing $\mathrm{s}_{1}$ and $\mathrm{s}_{2}$ as parts.

But, and here is the crux, it is also the case that any situation containing $\mathrm{s}_{1}$ and $\mathrm{s}_{3}$ will lack a truth-value for both $\mathrm{p}$ and $\mathrm{q}$. Why is this? It is because $\mathrm{s}_{3}$ precludes $\mathrm{p}$ ( $\mathrm{p}$ cannot be true with $\mathrm{s}_{3}$ 's contents) and $\mathrm{s}_{1}$ precludes $\mathrm{q}$. What is distinctive about this case is that $s_{3}$ doesn't preclude $\mathrm{p}$ by $\mathrm{p}$ being false in $\mathrm{s}_{3}$. Rather, $\mathrm{s}_{3}$ precludes $\mathrm{p}$ because, given the contents of $s_{3}$, p cannot have either truth-value. This is unlike the typical cases of metaphysical indeterminacy above, and captures the deep indeterminacy. So, in general any situation containing one of the pair $s_{1} / s_{2}$ and one of $s_{3} / s_{4}$ will not receive a truth-value for either $\mathrm{p}$ or $\mathrm{q}$. Thus the deeply indeterminate case is also captured: given the Kochen-Specker result, $\mathrm{p}$ and $\mathrm{q}$ cannot both receive truth-values in any situation and our interpretation is able to encode such constraints. In effect, what Kochen-Specker tells us is that $\mathrm{p}$ and $\mathrm{q}$ act as precluders for one another, making any situation containing parts in which both receive truth-values overdetermined in the relevant sense. Persistence therefore fails in these scenarios.

To summarise: what it means for something to be metaphysically indeterminate is for a number of different, incompatible situations to be real candidates for the

\footnotetext{
18 Suppose we fuse the minimal situation that Jones is $\mathrm{F}$ with the minimal situation that Jones is not $\mathrm{F}$. Call this situation s*. From what we have said, it is clear that neither 'Jones is F' nor 'Jones is not F' will be true in $\mathrm{s}^{*}$. Will any proposition be true in $\mathrm{s}^{*}$ ? Yes: it is true in $\mathrm{s}^{*}$ that it has a part in which Jones is $\mathrm{F}$, and likewise true that it has a part in which Jones is not $\mathrm{F}$.

What about the disjunctive proposition 'Jones is F or Jones is not F'? There is a choice-point here on the semantics: it could be true, false or neither true nor false. As it doesn't affect our main argument, we won't settle this question. Nevertheless it is interesting to note that, if such disjunctions are true in all cases like this, our model may be able to uphold excluded middle (though there's further work to do to show this).

Note, too, that the mereological relations between situations cannot be defined in terms of the mereological relations between the sets of propositions true in them. The set of propositions true in $s^{*}$ is not a fusion of the propositions true in the minimal situations in which Jones is $\mathrm{F}$ and not $\mathrm{F}$ precisely because persistence is not universal. This also has interesting consequences for the identity conditions of situations, which we cannot go into here.

Thanks to a reviewer for prompting us to think about this further.
} 
way the world is. A proposition is (metaphysically) indeterminate when it is true in some situation which is a candidate for actuality and false in another such situation. There is thus fundamental disagreement between the situations which are candidates for actuality. Situations allow unrestricted and unique fusion, so any situations can be combined. Furthermore, if two situations are candidates for actuality then their fusion is as well. This means there are situations which are candidates for actuality containing as parts situations which fundamentally disagree. If we accepted universal persistence, such situations would cause problems. But we do not: propositions persist only under restricted conditions. They fail to persist when overdetermined. This overdetermination can be expressed as preclusion: a proposition is overdetermined in a situation when that situation contains a part which precludes the proposition. A proposition is precluded by a situation when it cannot be true with the contents of that situation. Therefore, in situations which contain fundamentally disagreeing parts there are truth-value gaps concerning the propositions which are overdetermined by those fundamentally disagreeing parts. In typical cases of metaphysical indeterminacy, the propositions are precluded by virtue of being true in some part of the larger situation and false in some other part of that situation. By ruling out persistence, we avoid contradiction in these examples. Cases of deep indeterminacy are special cases of overdetermination where constraints are placed on which pairs of propositions can be true together. In these cases, the propositions are precluded by virtue of being true in some part of the larger situation and unable to have a truth-value in some other part of that situation. Each of the pair then lacks a truthvalue in combined situations. So there are no situations in which either a contradiction is true or the constraints imposed by deep indeterminacy are violated.

Thus we can safely hold that there is the fundamental disagreement required between candidates for actuality without either falling into contradiction or failing to capture deep indeterminacy.

\subsection{Application to other quantum cases}

In our discussion, we have so far focused on a particular quantum mechanical case where the Kochen-Specker theorem tells us that members of certain pairs of propositions cannot both receive definite truth-values. ${ }^{19}$ This is the issue of deep indeterminacy, and the example is one used in the literature to prosecute the BW model. In a recent paper, however, Calosi and Wilson have expanded the range of quantum mechanical cases that have been considered as instances of metaphysical indeterminacy. Specifically, the example we have discussed is a case of 'incompatible observables' in their terminology, and the further examples are those of superposition and entanglement (see Calosi and Wilson 2018, sect. 2.2). In this section, therefore, we wish to briefly show that the same mechanism we have used for the incompatible observables case can be applied to these other two examples where, arguably, quantum theory might warrant metaphysical indeterminacy. (We do so while still

19 This section was prompted by a request from a reviewer, to whom we are grateful. 
remaining agnostic on the value of quantum theory as a motivation for metaphysical indeterminacy as outlined in our Sect. 3.)

\subsubsection{Superposition}

The classic example of superposition is Schrödinger's unfortunate cat. The cat is put in a sealed box that has probabilistic mechanism (typically decay of a radioactive substance) which will release deadly gas if triggered. The system can be set up so that at some particular time the probability of the cat being alive and probability of the cat being dead are equal. The quantum mechanical upshot is that the cat is in a superposition of alive and dead. This superposition, given the interpretative assumptions we've been granting, is plausibly a related but distinct example of metaphysical indeterminacy: it seems metaphysically indeterminate whether the cat is alive or dead.

Our model can deal with this case in a natural way. Consider the following three situations, each of which is a candidate for actuality: $s_{1}$, a situation in which the cat is alive, $s_{2}$, a situation in which the cat is dead, and $s_{3}$, the fusion of $s_{1}$ and $s_{2}$. In $s_{1}$, it is true that the cat is alive and false that it is dead. In $s_{2}$ it is true that the cat is dead and false that it is alive. So, what status do these propositions have? According to our definition they are indeterminate, because there is a situation which is a candidate for actuality in which they are true and another situation which is a candidate for actuality in which they are false.

What is the case in $s_{3}$ ? It seems $s_{3}$ is overdetermined with respect to the cat's mortality, as parts of $s_{3}$ disagree about whether the cat is alive. Specifically, $s_{2}$ precludes the proposition that the cat is alive and $s_{1}$ precludes the proposition that the cat is dead. So, following our argument above, the proposition that the cat is alive is neither true nor false in $s_{3}$ (as is the proposition that the cat is dead).

The indeterminacy involved in superposition is therefore dealt with. In fact, it operates in just the same way as a garden-variety case of metaphysical indeterminacy, like the example of Jones's being F.

\subsubsection{Entanglement}

The phenomenon of quantum entanglement is more complicated on our picture. Entanglement is a feature exhibited by quantum systems when a composite system has definite values while the components of the system do not. For instance, consider two particles each in a superposition of two different properties (spin-up and spin-down in some direction, say). These particles can be prepared such that there is a perfect anti-correlation between their properties: if the first is measured and has one property, the other will always and immediately have the contrary property. If one is spin-up in the appropriate direction, the other is spin-down. This is so even though, because of the superposition, it is indeterminate which properties the particles have before measurement. More generally, the system composed of the two particles is definitely one in which both properties are had, but it isn't determinate which particle has which property. The composite system is determinate in a way that the parts of the system are not. 
Entanglement is a phenomenon of significant philosophical interest. It challenges background assumptions used in forming physical theories, assumptions such as separability (the idea that the state of a whole is summed from the state of its parts) and locality (the denial of action at a distance). From our point of view, what is relevant is the way that entanglement, once again given interpretative assumptions, gives a pattern of determinacy and indeterminacy which is distinct from both cases we have considered so far. In an interesting way, entanglement seems to be the inverse of deep indeterminacy. Deep indeterminacy is a constraint which prevents certain things being determinate together. Entanglement is a phenomenon by which two things must become determinate together.

To model this, we start as usual with some situations which are candidates for actuality. Take $s_{1}$ and $s_{2}$ to be the situations in which the first particle has spin-up and spin-down respectively in some direction. The particle is in a superposition of spin-up and spin-down, so as in the previous section the situation $s_{3}$ which fuses $s_{1}$ and $s_{2}$ will be one into which the relevant propositions don't persist. It is indeterminate whether the particle is spin-up or spin-down. The same is true of the second particle with corresponding situations $s_{4}-s_{6}$. What is special about entanglement is that the fusion of the superposed states produces some determinacy. Let $s_{7}$ be the fusion of $s_{3}$ and $s_{6}$. Although it is neither truth nor false in $s_{3}$ and $s_{6}$ that the particle in the situation is spin-up or spin-down, it is true in $s_{7}$ that one of the two particles is spin-up and the other is spin-down. Because this is true in $s_{7}$, it is true in a candidate for actuality (as $s_{7}$ is such a situation). It is false in no situation which is a candidate for actuality. Thus it is determinately true that one particle is spin-up and the other spin-down. However, for each particle it is indeterminate what spin it has. ${ }^{20}$

Our approach, therefore, allows us to capture the metaphysical indeterminacy (and determinacy) involved in entanglement by once more attending to what is the case in different situations which are all candidates for actuality. We have not, it must be admitted, explained why there is determinacy in $s_{7}$ which is not present in any sub-situation. But we don't take this to be the task, just as we haven't explained why the pairs of propositions in a deeply indeterminate case preclude definite values for one another. We are simply providing a consistent model for metaphysical indeterminacy which can accurately capture all the relevant claims.

(To be speculative for a moment: the holism seemingly involved in entanglement might make us reflect on which situations ought to be considered fundamental. It is tacitly assumed that smaller situations are more primitive, but there is nothing in

\footnotetext{
${ }^{20}$ In fn 18 we mentioned that there is a decision to be made in the semantics about the truth-value of a disjunction in a situation where both disjuncts are indeterminate through overdetermination. This choice will have an interesting connection to the explanation of the entanglement case. Suppose such disjunctions are true; the disjunction 'first particle spin-up or spin-down' will be true in $s_{3}\left(\right.$ and $\left.s_{7}\right)$. The disjunction 'second particle spin-up or spin-down' will be true in $\mathrm{s}_{6}\left(\right.$ and $\left.\mathrm{s}_{7}\right)$. In $\mathrm{s}_{7}$, where both disjunctions are true, the following true disjunction also emerges: '(first particle spin-up and second particle spin-down) or (first particle spin-down and second particle spin-up)'. What is ruled out is both particles having the same spin, i.e. the disjunction '(first particle spin-up and second particle spin-up) or (first particle spindown and second particle spin-down)'. The phenomenon of entanglement can then be expressed as the truth of one disjunction and the falsity of the other in $s_{7}$.
} 
the use of situations which requires this to be the case. A mereology of situations needn't take parts to be prior to wholes. Working out this thought, however, would take us far beyond the scope of this paper.)

This concludes our presentation of our model and interpretation. In what follows, we wish to evaluate the proposal. In particular, we will do so by looking at a nonexhaustive group of similar alternatives and showing a consequence our approach. In so doing, we also hope to clarify the view we've outlined.

\section{Evaluation}

We have offered a model which uses the basic insight of the Barnes-Williams account of metaphysical indeterminacy with some vital adjustments. These adjustments have some interesting effects. We'll briefly highlight one of these.

In our model, candidates for actuality can contain truth-value gaps. Thus the picture we present doesn't uphold classical logic and, in particular, rejects bivalence (which was a supposed virtue claimed by the BW model). ${ }^{21}$ It's a matter of debate whether we ought to uphold bivalence in contexts of metaphysical indeterminacy, but it is worth pointing out its denial already appears by our use of (typically partial) situations and our choice to take some propositions to be neither true nor false in some situations. Bivalence is not a feature well-suited to situation theory as we construe it.

Nevertheless, the model we provide of metaphysical indeterminacy involves a more dramatic rejection of classical logic than that implied just by use of situations. We have truth-value gaps even in candidate situations which are intuitively 'worldsized'. Further, we take it that a proposition can be neither true nor false in a situation even when that proposition is true or false in a part of that situation. This is precisely what happens in cases of metaphysical indeterminacy. On our picture, situations can fail to return a truth-value for a proposition both by underdetermining it (e.g. by not containing the relevant individuals) but also by overdetermining it (e.g. by containing parts which disagree about how things are). This leads to situations where propositions are neither true nor false even though all the relevant entities are in those situations. Some may find this feature off-putting; here we can only make it explicit. $^{22}$

If this feature is considered to be off-putting, this might motivate a search for alternatives. We do not claim that ours is the only way in which one could adapt the Barnes-Williams model to handle deep indeterminacy. But it is also worth indicating some challenges for a few of the alternatives which immediately present themselves. $^{23}$

\footnotetext{
21 It may also reject excluded middle, depending on the semantics. See fn 18.

22 Pickup (2016) adds to a cumulative case for taking such a view seriously.

23 We cannot consider all alternatives, but there are a few possible views we want to explicitly note we won't be discussing. The first is to use our structure but retain universal persistence, ending up with truth-value gluts rather than truth-value gaps. The second is to use the BW model and allow physically impossible but metaphysically possible worlds to be candidate precisifications.
} 


\subsection{Persistence as worldly}

One initially attractive variant on our view can be fairly quickly dealt with. This variant suggests a particular way to constrain persistence. It restricts persistence to cases where the relevant situations are both parts of the same world. To be explicit: on this view a proposition which is true in a situation is true in all extensions of that situation which are part of the same world.

It is worth first seeing why this is supposed to help, before showing why it doesn't. The garden-variety cases of metaphysical indeterminacy, like Jones's being or not being $\mathrm{F}$, threatened to lead to contradiction on our model if we allowed universal persistence, because there would be situations which are candidates for actuality which contained parts maintaining both that Jones is F and that Jones is not F. The condition that propositions only persist when the situations related by parthood are world-mates would deal with this issue. For the situations in which Jones is $\mathrm{F}$ and the situations in which Jones is not $\mathrm{F}$ are plausibly not jointly parts of any world (though they are jointly parts of some trans-world situations). So the condition would avoid the risk of contradiction in garden-variety cases of metaphysical indeterminacy. ${ }^{24}$

Unfortunately, though, this proposal has no such success in cases of deep indeterminacy. Deep indeterminacy tells us that there are some scenarios where pairs of propositions cannot both be given truth-values even when they are not contradictory. In the quantum-mechanical case we've discussed, $\mathrm{p}$ and $\mathrm{q}$ cannot both get truthvalues in any situation which is a candidate for actuality. But it seems the situation $s_{1}$ in which $p$ is true and the situation $s_{3}$ in which $q$ is true can both be part of the same world (if not, we need to be told why). Therefore, according to the condition provided, situations containing both $\mathrm{s}_{1}$ and $\mathrm{s}_{3}$ as parts will have $\mathrm{p}$ and $\mathrm{q}$ both persist into them. This is precisely the problem with BW's model that we started with: reality cannot be completely precisified into world-sized candidates for actuality.

A condition on persistence which allows persistence whenever situations are world-mates collapses into the original BW model. It is worlds, not situations, which are doing the work on such a view. It is worth remembering that we are framing the issue in terms of the persistence of propositions, rather than in terms of composition or candidacy for actuality. But the point remains the same in these other terms. If we restrict composition so that situations only compose a further situation when

\footnotetext{
Footnote 23 (continued)

Without going into detail, we find it hard with the first view to avoid falling into dialetheism and the second takes physically impossible worlds as candidates for actuality, which pushes against the idea that actuality implies physical possibility.

The proposals of Torza (2017) is more promising, though it departs from BW in a more radical way than we do. Wilson (2013, 2016; Calosi and Wilson 2018) offers a quite different approach which again is more promising. But we don't have space to survey all these alternatives here.

24 This restriction might be best expressed as a restriction on candidacy for actuality: if two situations which are candidates for actuality are fused, it is always and only the case that the fusion is a candidate for actuality if the component situations are jointly part of some world. As noted in the main text, deep indeterminacy shows this restriction too permissive.
} 
they are world-mates, ${ }^{25}$ or restrict candidacy for actuality so that situations are only candidates for actuality when all their parts are world-mates, we'll still be left with the problem of deep indeterminacy. Therefore, for the solution to work, we need persistence (or composition, or candidacy for actuality) to fail even within worlds.

\subsection{Imprecise worlds}

In his paper criticising the BW model for its failure to capture deep indeterminacy, Brad Skow briefly considers a tweak to their model which bears some resemblance to our approach. He says:

The orthodox interpretation of quantum mechanics is compatible with the existence of partial precisifications of reality. But the prospects of using this notion to put together a model of metaphysical indeterminacy similar to Barnes and Williams' model are dim. For suppose we keep their framework but replace perfectly precise possible worlds with imprecise possible worlds (sets of sentences from a language which suffers from semantic indeterminacy). Even when there is no metaphysical indeterminacy, we can expect it to happen that several imprecise possible worlds do not determinately misrepresent reality. So using imprecise worlds would give multiple actuality even when there is no metaphysical indeterminacy.

(Skow 2010, p. 858)

It isn't completely clear to us how to unpack what Skow means here. ${ }^{26}$ But he seems to identify something like the model we have presented. It is, however, importantly distinct. We developed our model from what we called the 'obvious' idea that lessthan-complete precisifications are substituted for BW's complete, precise worlds. This idea admits of two readings: an ordinary possible world is maximal, in the sense of treating everything, and precise, in the sense that everything that it does treat, it treats precisely. A situation is less than maximal, treating some propositions but remaining silent about others; there is nothing inherently imprecise about this. Our view uses such precise (but incomplete) situations to model indeterminacy. Skow here appears to consider the other reading: his imprecise possible worlds are maximal (although he is not explicit about this) but imprecise.

We are inclined to agree that imprecise worlds would not save the Barnes-Williams model, as it would over-generate indeterminacy. If we are using imprecise things in the modelling, even a precise reality would appear indeterminate. But, of course, that is not what we are doing. We use non-maximal but precise situations to model indeterminacy, and give circumstances when fusing precise situations leads to

\footnotetext{
25 A specific version of this view follows from one way to set up situation theory. Some situation theorists only allow actual situations to exist. If so, it is only actual situation that can be composed, which are trivially world-mates in typical contexts. In this context, where we are considering multiple actualities, this situation-theoretic choice would be unwise.

${ }^{26}$ In particular, it isn't clear exactly what sets of sentences from a language suffering from semantic indeterminacy has to do with the imprecision of possible worlds.
} 
truth-value gaps. In cases where there is no metaphysical indeterminacy, there will be no gaps in the fusions. Our model therefore doesn't over-generate indeterminacy. So, in effect our proposal is simply that Skow considers the wrong modification: don't use complete but imprecise worlds, use incomplete but precise situations. ${ }^{27}$

\subsection{Primitive indeterminacy of parthood}

A final alternative we'll consider retains much of our model but makes an important change. It uses situations, as incomplete precisifications, but takes indeterminacy to be a matter of unsettledness about which situations are parts of the actual world. This offers a view with more surface resemblance to the original BW model, but there are reasons to be wary of it. In the first place, there are significant departures from BW once the theory is more closely examined, and secondly, we are worried that the phenomenon of deep indeterminacy is not adequately captured.

But we are getting ahead of ourselves. First, the alternative. Return to the example above of our quantum system in situations $s_{1}-s_{4}$ : $p$ is true in $s_{1}, \neg p$ is true in $s_{2}$, $q$ is true in $s_{3}$ and $\neg q$ is true in $s_{4}$. But $s_{1}-s_{4}$ don't give a truth-value for either of the propositions from the other pair. There are also situations which have more than one of $s_{1}-s_{4}$ as parts: these are the situations into which, on our model, the truth-values do not persist. On our model, $\mathrm{p}$ and q receive no truth-value in these situations.

Recall that we have presented our view using the framing of a semantic restriction on when propositions persist. The alternative we now wish to consider is most easily expressed by instead considering a mereological restriction on which situations are able to compose a further situation. This won't matter to the formal structure of the alternative but makes exposition easier. ${ }^{28}$

In these terms, this alternative states that there is some situation $\mathrm{S}$ which is the actual world. All of $s_{1}-s_{4}$ are candidates for actuality. But it is indeterminate which of each pair $s_{1}-s_{2}$ and $s_{3}-s_{4}$ are parts of $S$. In other words, it is indeterminate which of $\mathrm{s}_{1}-\mathrm{s}_{4}$ compose $\mathrm{S}$. There is thus primitive indeterminacy in the parthood relations of reality: in BW's terms we might say that it is unsettled which of each pair of situations is part of the actual world. (BW of course have unsettledness in a different form as primitive in their model, hence the abandonment of reductive ambitions.) The parthood relations are indeterminate but universal persistence need not be denied. All of $\mathrm{s}_{1}-\mathrm{s}_{4}$ remain candidates for actuality, but $\mathrm{S}$ contains only some of them (and it is indeterminate which). The situations which are parts of S are the

\footnotetext{
27 Calosi and Wilson (2018) sect. 3.4.2 provides further argument against a partial precisifications view of the form that Skow envisages. One worry is about saving classical logic, and we concede our precise but incomplete situations don't do this. The other worry is that the indeterminacy of imprecise worlds will need to be explained by some other means, which doesn't arise for our view for the reason that we don't have imprecise worlds, but precise situations.

${ }^{28}$ To be explicit: in terms of restricted persistence, the alternative spelled out below takes which propositions persist to be itself indeterminate. Some propositions do persist from $\mathrm{s}_{1}-\mathrm{s}_{4}$ to their extensions, it is just indeterminate which. Thus either $\mathrm{p}$ or $\neg \mathrm{p}$ and either $\mathrm{q}$ or $\neg q$ persist, though it isn't determinate which. Contrast this with our view, on which none of these persist.
} 
ones whose propositions are true in S. Because its parts are indeterminate, it is indeterminate which propositions are true in $\mathrm{S}$.

Why not accept this alternative? We are concerned that the way that it deals with deep indeterminacy is inadequate. Let's make clear why.

Though this view doesn't require that the propositions true in each of $s_{1}-s_{4}$ are all true in $S$, it does tell us that one from each pair of situations $s_{1} / s_{2}$ and $s_{3} / s_{4}$ is part of $\mathrm{S} .{ }^{29}$ In other words, though it is unsettled whether $\mathrm{s}_{1}$ or $\mathrm{s}_{2}$ is part of $\mathrm{S}$ (and likewise for $s_{3}$ and $s_{4}$ ), because the parthood relations are indeterminate, it is determinate that either $s_{1}$ or $s_{2}$ is part of $\mathrm{S}$. Likewise, it is determinate that either $s_{3}$ or $s_{4}$ is part of $\mathrm{S}$. Universal persistence holds in this context, so what is true in a part of a situation is true in a whole situation. If it is determinate that either $s_{1}$ or $s_{2}$ is part of $S$, then it is determinate that either $\mathrm{p}$ or $\neg \mathrm{p}$ is true in $\mathrm{S}$ (though of course it is indeterminate which) and it is determinate that $\mathrm{q}$ or $\neg \mathrm{q}$ is true in $\mathrm{S}$ (though indeterminate which). The propositions persist, though which propositions is not determinate because which situations are parts of $\mathrm{S}$ is not determinate. ${ }^{30}$ Thus in $\mathrm{S}$ while it is indeterminate whether $\mathrm{p}$ and indeterminate whether $\mathrm{q}$, it is determinate that one of each pair is true.

This is already a violation of the spirit of Kochen-Specker and similar 'no-go' theorems in quantum mechanics: the point of such theorems is that certain pairs of propositions cannot be true together. S contains the sort of conjunctions of propositions that are supposed to be ruled out. But violating the spirit of a constraint is not a particularly effective criticism of a model, so we wish to spell out two reasons that we prefer our own account to the primitive indeterminacy of parthood model.

In the first place, there are (contested) ways that a violation of the letter of Kochen-Specker can be derived. These are contested because they rely on assumptions which are themselves controversial in the context of indeterminacy and associated non-classical logics. But given that BW wish to preserve classical logic and the fact that surface resemblance to the BW might be a motivation for the view, it is instructive nevertheless to spell these out. ${ }^{31}$

The view we're considering suggests that, in $S$, it is indeterminate whether $p$, indeterminate whether $\neg$ p, indeterminate whether $q$ and indeterminate whether $\neg q$. It is, however, determinate that ( $p \vee \neg p)$ and determinate that $(q \vee \neg q)$. If we add the very natural claim that determinacy is closed under conjunction, we can conclude that it's determinate that $[(p \vee \neg p) \wedge(q \vee \neg q)]$.

To get an explicit violation of Kochen-Specker, though, we need both $\mathrm{p}$ and $\mathrm{q}$ to get determinate truth-values in a situation. One way do so is to first add the assumption that conjunction distributes over disjunction, whereby we get the unwieldy claim: it is determinate that $[(\mathrm{p} \wedge \mathrm{q}) \vee(\neg \mathrm{p} \wedge \mathrm{q}) \vee(\mathrm{p} \wedge \neg \mathrm{q}) \vee(\neg \mathrm{p} \wedge \neg \mathrm{q})]$. If we

\footnotetext{
29 Recall that we are ignoring z-direction for simplicity: strictly speaking there would be a third pair of situations which gave determinate values for $\mathrm{z}$-direction too.

${ }^{30}$ In the terminology of restricting persistence rather than composition the view says it is indeterminate which of the pairs $s_{1} / s_{2}$ and $s_{3} / s_{4}$ is such that what is true in them persists to a situation containing all of these as parts. But the truths from one of each pair certainly do persist.

31 Thanks to a referee for pushing us to consider these assumptions.
} 
further take it that a disjunction being determinate implies that it is determinate that a disjunct is true, we arrive at a violation. For we can conclude that either $(p \wedge q)$ or $(\neg p \wedge q)$ or $(p \wedge \neg q)$ or $(\neg p \wedge \neg q)$ is determinately the case. All of these are ruled out by Kochen-Specker.

Of course, the assumptions used here can and will be challenged. ${ }^{32}$ But challenging them would require giving up classical logic, which BW do not wish to do. Now, we give up classical logic, so have no disagreement in principle with this move. It is simply worth noting that this alternative is not clearly closer to BW than our proposed model, and that there will be work to do to block a reappearance of the deep indeterminacy issue.

The second worry we have with the model also concerns its approach to deep indeterminacy, but from a different direction. If $\mathrm{S}$ contains one of $\mathrm{s}_{1}$ or $\mathrm{s}_{2}$, but not both, and one of $s_{3}$ and $s_{4}$, but not both, then deep indeterminacy is captured using a different structure to garden-variety indeterminacy. This is because in garden-variety indeterminacy only one of a pair of conflicting situations is part of S (i.e. only $\mathrm{s}_{1}$ or $\mathrm{s}_{2}$ but not both). While in a deeply indeterminate case conflicting situations are both parts of $S$, because $s_{1}$ (and $s_{2}$ ) conflicts with both $s_{3}$ and $s_{4}$. In itself, this might be considered a virtue, as deep indeterminacy is a distinct phenomenon from gardenvariety indeterminacy. But the issue is that the model thereby loses the ability to represent the distinctive relationship between the pairs of situations which cannot be jointly determinate.

Consider another example, where two independent propositions are both indeterminate. Suppose it is indeterminate whether Jones is F and, separately and independently, indeterminate whether Smith is G. The model we are considering will treat this in precisely the same way as deep indeterminacy. There will be primitive indeterminacy in whether the world has as a part a situation in which Jones is F or a situation in which Jones is not F; likewise with a situation in which Smith is G and one in which she isn't. This means that it will be determinate that [(Jones is $F \vee \neg$ Jones is $F$ ) $\wedge$ (Smith is $G \vee \neg$ Smith is $G$ )]. But, as described in the previous paragraphs, avoiding a violation of Kochen-Specker will require rejecting assumptions that warrant the move from this to (Jones is $F \wedge$ Smith is G) or ( $\neg$ Jones is $F \wedge$ Smith is G) or .... Thus a case of deep indeterminacy and a case of independent indeterminacy are represented the same way, which undermines the expressive power of the account we're currently considering.

In our view, and this is somewhat speculative, it is the fact that the model permits the determinate conjunction of $[(p \vee \neg p) \wedge(q \vee \neg q)]$ which is the root of the problem. As much as revising classical logic might prevent this from leading to a direct violation of Kochen-Specker, a natural way to capture Kochen-Specker within a model of indeterminacy is to rule out precisely such conjunctions in the appropriate

\footnotetext{
32 See, in particular, Torza (2017) for a detailed discussion of a way to capture deep indeterminacy which denies compositionality.
} 
cases. So we do not think that, ultimately, this model can adequately capture deep indeterminacy. ${ }^{33}$

To conclude this section, we can express some of these misgivings in the terminology of BW's One True world. According to the current model there is One True actual world, but it is unsettled which situations it has as parts. The parts of the One True world, however, are fully determinate, and universal persistence obtains. So, whichever parts these may be (and this is indeterminate), the propositions true in them are true in the One True world. It is as though reality is poised between alternative situations as its parts, but in each of these alternatives we have members from both pairs of propositions.

But the point of deep indeterminacy is that it tells us that the sort of things reality is poised between are not possible: there can't be One True world with a truth value for $\mathrm{p}$ and a truth value for $\mathrm{q}$. Being poised between impossible alternatives, plausibly, is itself impossible. If no alternative is possible, then the One True world isn't possible either.

This leads towards a surprising result. It is not the fact that the BW model uses complete precisifications that is the issue; incomplete precisifications (situations) can likewise lead to a violation of Kochen-Specker type considerations if we accept that there is One True world, even if it is not determinate which it is. For this world, if it is precise, will not be able to accurately represent reality. Instead, we need to do away with the One True world: it is not that it is indeterminate which is the One True world, but that the One True world itself is not determinate. The One True world has incompatible parts.

\subsection{Evaluation summary}

In this section, we have brought out a consequence of the model we have presented and compared it with some alternatives. The consequence is that, if there is metaphysical indeterminacy, candidates for actuality have irreducible truth-value gaps. Thus the contenders for reality are not bivalent. This might seem undesirable but might be, we think, a consequence of taking metaphysical indeterminacy seriously.

The alternatives to our model we have considered seem to either (1) suffer from the same issues with deep indeterminacy that undermine the BW approach we started with (and in one case collapse into it) or (2) stipulate indeterminacy when there is none (as argued by Skow). This has hopefully made clearer exactly how our own model should be understood and given some support for it.

\footnotetext{
33 It could be suggested that only one of $\mathrm{s}_{1}-\mathrm{s}_{4}$ is a part of $\mathrm{S}$ (and it is indeterminate which) (thanks to Gonzalo Rodriguez-Pereyra for this suggestion). But this seems difficult to square with claims about fixed relative values of variables in quantum mechanical cases (see Torza 2017). It also seems to give up the desiderata that there is One True (complete) actual world: such a world would be incomplete.
} 


\section{Conclusion}

We have constructed a model of metaphysical indeterminacy that can handle deep indeterminacy. After outlining Barnes and Williams's recent meta-level model of metaphysical indeterminacy, we rehearsed arguments that it is incompatible with the kind of 'deep' indeterminacy that is arguably involved in quantum mechanics, and in any case ought to be handled by a model of metaphysical indeterminacy. This might raise doubts that a meta-level account can make space for such indeterminacy. We believe it can, and so set out to give a reformulation of the Barnes-Williams model using incomplete, local situations. We explained the basic ideas behind situation semantics and showed how a domain of incomplete situations, together with a mereology defined on them, can give rise to a variant of the Barnes-Williams model that is close to the original but accommodates deep indeterminacy. The core idea is that the candidate precisifications are precise but non-maximal: these precisifications are parts of the world. Indeterminacy is then a matter of something being true in some candidate situations and false in others. This leads to incompatibility but not, we argue, contradiction. The manoeuvres required to retain incompatibility without contradiction give an interesting account. Finally, we evaluated our model to show how it is to be understood and how it is preferable to similar alternatives.

Our conclusion is that there is at least one viable meta-level account of metaphysical indeterminacy which can handle deep indeterminacy. ${ }^{34}$

Open Access This article is distributed under the terms of the Creative Commons Attribution 4.0 International License (http://creativecommons.org/licenses/by/4.0/), which permits unrestricted use, distribution, and reproduction in any medium, provided you give appropriate credit to the original author(s) and the source, provide a link to the Creative Commons license, and indicate if changes were made.

\section{References}

Akiba, K. (2004). Vagueness in the world. Nôus, 38(3), 407-429.

Austin, J. L. (1950). Truth. Proceedings of the Aristotelian Society, 24, 111-128.

Barnes, E. (2010). Ontic vagueness: A guide for the perplexed. Nôus, 44(4), 601-627.

Barnes, E., \& Williams, J. R. G. (2011). A theory of metaphysical indeterminacy. Oxford Studies in Metaphysics, 6, 103-148.

Barwise, J. (Ed.). (1988). Notes on branch points in a situation theory. In J. Barwise (Ed.), The situation in logic. Stanford: CSLI.

Barwise, J., \& Etchemendy, J. (1987). The liar: An essay in truth and circularity. Oxford: OUP.

Bokulich, A. (2014). Metaphysical indeterminacy, properties, and quantum theory. Res Philosophica, 91(3), 449-475.

Calosi, C., \& Wilson, J. (2018). Quantum metaphysical indeterminacy. Philosophical Studies. https://doi. org/10.1007/s11098-018-1143-2.

\footnotetext{
${ }^{34}$ We are grateful to a number of people for comments on earlier drafts of this paper, including: two excellent referees for this journal; audiences at the Metaphysics of Entanglement work in progress group, the Oriel Philosophy Group, and the Epistemology of Metaphysics workshop 1, Helsinki; David Glick, Nick Jones, Gonzalo Rodriguez-Pereyra and, especially, Dani Kodaj (who was a co-author on the initial draft). The original idea for this paper was conceived while we were both postdoctoral fellows on the Metaphysics of Entanglement research project in Oxford, funded by the Templeton World Charity Foundation.
} 
Darby, G. (2010). Quantum mechanics and metaphysical indeterminacy. Australasian Journal of Philosophy, 88(2), 227-245.

Darby, G., Pickup, M., \& Robson, J. (2017). Deep indeterminacy in physics and fiction. In O. Bueno, G. Darby, S. French, \& D. Rickles (Eds.), Thinking about science, reflecting on art. London: Routledge.

Edgington, D. (1985). The paradox of knowability. Mind, 94, 557-568.

Elbourne, P. (2005). Situations and individuals. Cambridge, MA: MIT Press.

Elbourne, P. (2013). Definite descriptions. Oxford: OUP.

Fine, K. (Ed.). (2005). Tense and reality. In Modality and tense. Oxford: OUP.

Fine, K. (2017). Truthmaker semantics. In B. Hale, C. Wright, \& A. Miller (Eds.), A companion to the philosophy of language. Oxford: Blackwell.

Glick, D. (2017). Against quantum indeterminacy. Thought, 6(3), 204-213.

Kratzer, A. (1989). An investigation of the lumps of thought. Linguistics and Philosophy, 12(5), 607-653.

Kratzer, A. (2017). Situations in natural language semantics. In E. N. Zalta (Ed.) Stanford Encyclopedia of philosophy (Winter 2017 Edition). https://plato.stanford.edu/archives/win2017/entries/situations -semantics/.

Pickup, M. (2016). A situationalist solution to the Ship of Theseus puzzle. Erkenntnis, 81(5), 973-992.

Rumfitt, I. (2015). The boundary stones of thought: An essay in the philosophy of logic. Oxford: OUP.

Skow, B. (2010). Deep metaphysical indeterminacy. The Philosophical Quarterly, 60, 851-858.

Torza, A. (2017). Quantum metaphysical indeterminacy and worldly incompleteness. Synthese. https:// doi.org/10.1007/s11229-017-1581-y.

Williams, J. R. G. (2008). Ontic vagueness and metaphysical indeterminacy. Philosophy Compass, 3(4), 763-788.

Wilson, J. (2013). A determinable-based account of metaphysical indeterminacy. Inquiry, 56(4), 359-385.

Wilson, J. (2016). Are there indeterminate states of affairs? Yes. In E. Barnes (Ed.), Current controversies in metaphysics. London: Taylor and Francis.

Yablo, S. (2014). Aboutness. Princeton, NJ: Princeton University Press. 